\title{
RISK OF TOXOPLASMOSIS FROM CATS
}

\author{
WASAN AMJAD ALOBAIDII; MANAL HAMMADI HASAN AND \\ BAYDAA YOUNIS MAHMMOOD
}

Department of Microbiology, College of Veterinary Medicine University of Mosul, Mosul, Iraq

Received: 12 July 2020; $\quad$ Accepted: 31 July 2020

\begin{abstract}
Toxoplasmosis is a zoonotic disease caused by Toxoplasma gondii, there are two hosts, cats play an important role as definitive hosts , cattle, sheep ,goats and human may play as intermediate hosts. Humans be infected by eating tissue cysts from Not cooked flesh and eat contaminated feed or water with oocytes shed in the faecal of infected cats. diagnosis of $\mathrm{T}$. gondii itself is affirmation the infection but difficult, so many laboratories use serological methods to detect immunoglobulins which specific to $\mathrm{T}$. gondii : latex agglutination (LA) test, Enzyme Linked Immuno Sorbent Assay and indirect fluorescent antibody test (IFAT) those methods was differ in specificity and sensitivity there is no systematic review that can perform the state of toxoplasmosis in the definitive host. Therefore the aims of the current existing review was to decided the risk factor of $\mathrm{T}$. gondii infection and demonstrated the epidemiological form of infection in cats in Iraq.
\end{abstract}

Key words: Cat, Toxoplasmosis, Risk, Epidemiology, host.

\section{INTRODUCTION}

Toxoplasma gondii is an obligatory intracellular parasite, belongs to the phylum Apicomplexa, T.gondii infects warmblooded animals (Flegr, 2013).

Cats play critical role for $\mathrm{T}$. gondii as final hosts, and definitive hosts that reproduce oocytes in their faecal, which contaminate sand, feed and water (Cenci-Goga et al., 2011). final host shed oocytes for a limited interval of time (7-14 days), up to 1000000 of oocytes possibly shed oocytes which stay as infective stage for up to one month and

Corresponding author: Wasan Amjad Alobaidii E-mail address: wasenamjad@yahoo.com Present address: Department of Microbiology, College of Veterinary Medicine University of Mosul, Mosul, Iraq resist to low temperature, dryness, and sensitive to heat, which destroyed at $70{ }^{\circ} \mathrm{C}$ for $10 \mathrm{~min}$ (Dubey and Jones, 2008)

Infection of definitive and intermediate hosts occurs through ingest of tissue cysts or oocytes. cats shed oocytes followed by ingest tissue cysts, a cat take it at least 1000 oocytes so as to improve an infection, taking it a one bradyzoite is sufficient for a cat to adequate to infection (Dubey et al., 2008)

Life cycle.

The life cycle of this parasite is intricacy, which include asexual stage of intermediate hosts and a sexual stage which occur in the definitive hosts (Edwards and Dubey, 2013).

Toxoplasma gondii has two-host (Fancourt and Jackson, 2014) .For T. gondii, cats can 
act as a definitive host (Dolores and Dubey, 2016), after the ingest of tissue cysts by the cats, organisms excyst as bradyzoites which penetrate the epithelium of intestine (Alonso Aguirre et al., 2019). Initiation of sexual reproduction which development into oocysts Within epithelial cells when bradyzoites transform into male and female gamonts. Toxoplasma oocytes are released into the intestinal lumen, and subsequently passed in faeces (Joanne, 2012)

Intermediate hosts are infected by ingest contaminated food, water or soil with oocytes/sporocysts (Hill and Dubey 2002). mammals is a intermediate hosts of $\mathrm{T}$. gondii, in the alimentary tract of the intermediate host, sporozoites excyst from oocysts/sporocysts and penetrate the intestinal epithelium. Sporozoites infect epithelial cells and develop to tachyzoites which multiply rapidly. infected cell with T.gondii tachyzoites cause cell lysis, then tachyzoites released which infect other epithelial cells The infection then distribute to other organisms through lymph and blood stream (Uzal et al., 2016). When the immune system suppressed the transformation of tachyzoites into bradyzoites and the development of tissue cysts. in muscle and neurological tissues, Cysts still in the organs of the intermediate host for life, but may reactivate if the host becomes immunosuppressed (Ferguson et al., 1989). The lifecycle is complete when the definitive host consumes tissue cysts from an intermediate host.

\section{Transmission}

Toxoplasma gondii is transmitted to cats by ingestion of infected tissue by bradyzoites in infected tissues or by consumption of oocytes.

There are four main routes of $\mathrm{T}$. gondii transmission to intermediate hosts occurs by:

* By the consumption of oocysts

* Congenital or vertical transmission (Parameswaran et al., 2009)
* horizontal transmission between intermediate hosts following the consumption of bradyzoites

* sexual transmission via bradyzoites in sperm (Dass et al., 2011).

consumption of contaminated food, water or soil with oocyte is the main source of infection in humans

\section{Clinical signs}

There are two forms of infection of Toxoplasma gondii, acute and chronic. Acute form results from the multiplication of tachyzoites in the intermediate host (active parasite) these form cause cell lysis, tissue necrosis and inflammation, then death occur (Muhammad et al., 2018).

Chronic form occurs when immunosuppression, tachyzoites was transform to intracellular cysts containing bradyzoites (parasite dormant). (Vyas 2015).

Clinical appearance of cats infective with $\mathrm{T}$. gondii comprise, depression, loss of appetite and increase body temperature, follow up by peritoneal effusion, jaundice, dyspnea, diarrhoea, weight loss, muscle hyperaesthesi, , paresis ,inflammation of iris and pancreas (Bethânia et al., 2014).

In cats, toxoplasmosis is intense in transplacentally infected kitten, which repeatedly promote hepatitis or cholangiohepatitis, pneumonia, and encephalitis and display signs of ascites, lethargy, and dyspnea.

The main symptoms of Toxoplasma gondii infection is pneumonia and acute respiratory signs Ocular toxoplasmosis occur in kitten and never appeared any clinical manifestation chorioretinitis, Aqueous flare, glaucoma, and retinal separation are mutual observed (Muhannad et al., 2018) (Dubey \& Beattie 1988).

\section{Epidemiology}

Cats have a critical role in the infection of toxoplasmosis, so expand about $\mathrm{T}$. gondii 
infection in cats is an importance. It is worth remanding that epidemiological inspection are still the useful method for evaluate the case of T. gondii infection (Chaichan, 2018) grazing herbivores would be expected to have a high risk of exposure to $\mathrm{T}$. gondii oocysts on pasture, a low risk of transplacental transmission (Parameswaran et al., 2009), no risk of transmission via carnivorism, and a low risk of sexual transmission (depending on the frequency of intercourse.( Kolören and Dubey, 2020).

There are a correlation between environment and definitive host which include density of definitive host which lead to increase in parasite shedding which contaminate environment. defecation behaviour of definitive host, (Dubey, et al., 2009).like Bury the faeces, may also increase the probability and participate to parasite transmission. The presence of definitive and intermediate hosts together in the same environment, not necessary, for a intermediate host to become infected. Similarly, both hosts have to present at the same environment, that increase the incidence of consumetion of bradyzoites by definitive host, lead to increase probability the infection of intermediate host (Huan et al., 2017).

So complete or partial separation in a distance between the two hosts lead to reduce the infection (Mulisa, 2014).

\section{Diagnosis}

Many types of direct and indirect methods used to expose $\mathrm{T}$. gondii infection or antiToxoplasma antibodies. several methods which are usable to investigate parasite (Anthea, 2015). include: histological coincidence, isolation of the organism in tissue culture, and detection of the parasite DNA by the polymerase chain reaction (PCR) or by a collection of these techniques to a quorate diagnosis, whereas the serological test are used to investigate the types of immunoglobulins (Mohammad et al., 2015) .

\section{Isolation of the parasite in laboratory animals}

The detection of T.gondii was attempt by the administration of suspected specimen which included blood, CS fluid, Lymphatic nodes or other body fluids or organs, in the peritoneal cavity of the immune-suppressed mice or BALB/c mice. in the peritoneal fluid tachyzoites was detected with microscopy within 1 weeks after inoculation. The parasite was detected after fixation the sample with methanol and stain with Wright, Giemsa then observed by a microscope (Janina et al., 2010).

\section{Propagation of parasite in Cellular culture}

Cellular culture was used to detection and segregation of parasite, the parasite has been isolated from lower respiratory system and CS fluid or diagnosis of ocular. To approved host-parasite interaction Cellular cultures are also can be used and used in inherent resistance, molecular characterization of Toxoplasma isolates, and for the valuation of vaccine and treatment development, after the inoculation of tissue culture with suspected samples, Cyto Pathic Effect was appear which manifested by demolition of the cellular layers and is concerning with the initial amount of tachyzoites in the specimen(Al-Saidya, 2006).

\section{Serological diagnosis}

The routine laboratory detection in both humans and animals based on many types of sero-diagnosis of specific immunoglobulins in serum and milk. The increase of the titer of antibodies are related to the immune response next the infection, the limitation the duration of infection by determination the type of immunoglobulin in humoral immune. (Karen et al., 2019).

Several types of serological tests used to measurement various kinds of antibodies, which record rise and decline during or next infection. The detection of the types of immunoglobulin immunoglobulin used in T. gondii serodiagnosis, including immunoglobulin's (G,A,M and E), the 
elevation of $\operatorname{IgM}$ mean the infection is early and relates to the manifestation of intense toxoplasmosis. Mostly immunoglobulin (M) can be expose in serum only 7 days after the infection (Majda, 2017).

IgM antibodies present for even 18 months in the maternal circulation after invasion effect, so the accuracy of this diagnostic process because determining whether an antibody is from active or prior infection is critical during gestation. If an antibody is from a prior infection, no sequel for the embryo normally occur. If the infection occur during pregnancy, the clinician have to decide on administer drugs to treatment to avoid disease complication (Al Hamada et al., 2019)

Detection of $\operatorname{IgG}$ antibodies help to diagnosis Immunoglobulin $(G)$ antibodies which showed within 1-2 weeks following infection, and reach high titer within 3060 days, then decline, but may persist lifelong (Silvia et al., 2005).

Arising the titer of immunoglobulin indicate prior infection., detection of immunoglobulin $(\mathrm{G})$ is used as diagnostic indicator and assist veterinarians to determine chronic infection or not. However, this antibody still has hardness in distinguishing prior infection from a new infection (Eman and Azhar, 2016).

There are several serological methods have been decided for the detection of anti $\mathrm{T}$. gondii immunoglobulin; these include Sabin-Feldman dye test (SFDT), In-direct Fluorescent Assay (IFA), latex agglutination tests, and Enzyme Linked Immuno-sorbent Assays (ELISAs) (Patrick, 2019).

\section{Molecular diagnosis}

Polymerase Chain Reaction is mutual Molecular diagnostic method using to detection of $\mathrm{T}$. gondii, it is a simple and sensitive method and suitable to applied in all clinical samples, otherwise PCR can be used for genetic characterization of the parasite (Daland, 2012).
Other best characters of PCR that this molecular method easily differentiates $T$. gondii from other cyst forming protozoa.

Molecular diagnostics method depend on isolation and detection of specific DNA sequences. (Falah, 2018)

\section{Vaccine}

There is one vaccine type "Toxovax" which depend on attenuated of S 48 strain which used to evade vertical transmission in sheep. This vaccine is give limited time of protection, expensive, and give some disadvantage effects, this vaccine not adequate for human use, because may reverse to a virulent isolates (Afsaneh et al., 2019).

Many vaccination trial versus T.gondii have been attempt which applied in animals models, including in-activated and attenuate vaccine, sub-unit vaccine, and DNA vaccines. The results have offer that it may be develop an affective vaccine against $\mathrm{T}$ gondii but not approved successfully in field. (Patrick, 2019).

\section{Treatment}

There is no confirmed treatment for clinical $\mathrm{T}$ gondii infection in cats. Therapy include sulphonamides, pyrimethamine, trimethoprim and clindamycin, spiromycin, either alone or together (Dabritz et al., 2007).

\section{Toxoplasmosis in Iraq}

Several studies was conducted T.gondii in Iraq, which include seroprevelance, isolation , and diagnoisis in different hosts , So we include some of these studies:

(Aiz, 2016; Kader and Khayat, 2013; AlDabagh et al., 2014; Eman and Azhar, 2016; Al Hamada et al., 2019; Nawzat, 2017; AlTaie and Shadan, 2011; Mikail and AlBarwary, 2014) These studies looked at the infection of small ruminates with T.gondii, other studies which include the infection in cattle and buffaloes (Sakban and Aiz, 2020; Al-Farwachi et al., 2008; Akber et al., 2004) 
other studies include the parasite in camels (Saad et al., 2019; Hanon, 2017) in dogs there are some studies conducted the parasite which include (Abass, 2013) other studies include the diagnosis of parasite in equidae (Alshahery and Mansour, 2012; Asal and Al Zubaidy, 2016).

\section{Studies dealing with toxoplasmosis in cats in Iraq are very few, and these studies have receded in several governorates which include the follow:}

(Dhamraa et al., 2014) which study the seroprevelance of feline toxoplasmosis in stray cats in Baghdad city, the researchers collect 50 blood samples from cats in different regions, age and genders, the survey was attempt using to investigate of specific toxoplasma IgG using ELISA test, the result showed that the $66 \%$ of stray cats was infected with Toxoplasma gondii, the female was recorded high prevelance of infection that male and the young kittens (2months of age) reported high prevelance than adults once, the researchers find the relationships between the infection of cats with T.gondii and warm and humid weathers of Iraq, the high seroprevalence of $\mathrm{T}$. gondii is due to hunting habit of stray cats that their diet includes rodents, placenta, stillborn foeti and wild birds .other samples in this study which include pieces from internal organs of these cats were taken for histopathological examination, the result of these samples revealed The intestine of animal expressed shizon in the epithelial cells surrounded by erosion lesion and these cyst also was seen in the LP of the intestine as well as inflammatory cells mainly eosinophils and neutrphils infiltration, while the liver showed large necrotic area with basophilic tachyzoits intracytoplasm of swelling hepatocyte, in addition to granulomatous lesion consisting from aggregation of macrophages and lymphocytes and zoites in sinusoids, It reported severe pathological lesions in the lung, intestine, liver and spleen of cats that expressed high level of OD antitoxoplasma IgG antibody.
Other researchers Alexandra et al. (2013), which study the presence of toxoplasma gondii antibodies on sera of 207 stray cats trapped as a result of increase population, stray cats were capture on military bases in Iraq (Baghdad) as department of the USA Army (Zoonotic Disease) Surveillances Program by using Latex Agglutination Test, the results showed that immunoglobulin against $\mathrm{T}$. gondii were detect in $30.4 \%$ of the cats, , the cats from west Iraq had a lower seroprevalence (than cats from North of Iraq and cats from Baghdad. (66.6\%) percentage of infection in Tom, without significant of variance was detected between sex and Toxoplasma positivity. the researchers interpret the increase percentage of infection in stray cats to occasion to victim of $\mathrm{T}$. gondii intermediate hosts such as rodent, or become infected through contaminated sand or water.

Other study attempt by Hemdad et al. (2019) in north of Iraq in Erbil city -Kurdistan Region, which include Geno-typing of $\mathrm{T}$ gondii from astray cats by using RELP Polymerase Chain Reaction, 100 blood specimen were collect from stray cats to detect the specific antibodies of $\mathrm{T}$ gondii using MAT test and 12 samples of heart from seropostive cats to Multi-locus Polymerase Chain Reaction-RFLP genotyping of $\mathrm{T}$. gondii, the results of study can be conclud that $\mathrm{T}$. gondii isolate from heart tissue of cat by 6 genetic markers (SAG1, GRA6, SAG3, BTUB, c22-8 and c29-2). The type III is also name as ToxoDB genotype \#2.

Other study was done by Ziadoon et al. (2017), to detection of specific antibodies of $\mathrm{T}$. gondii in different hosts (450 women, 124ewes and 79 does , 20 stray cats, 28 rabbits and 28 birds) in DIYALA PROVINCE by using ELISA and Latex agglutination test, the results showed that $50 \%$ of cats was infected with T.gondii by using both tests, the researchers Proved this results to habit lives of which rove free and often form groups of animals that freshen 
enclose proximately to humans and other hosts .

Afkar and Azhar 2014 investigate the most important protozoa in 80 feces of stray cats in Baghdad using routine fecal examination, the results showed that $3.75 \%$ of cats infect with T. gondii.

The most important review which attempt by Saleem (2007) which included the most important microorganism which recorded in dogs and cats in Iraq, T. gondii recorded in 11 Laboratory confirmation and reference in cats.

\section{ACKNOWLEDGMENT}

The authors are very grateful to the University of Mosul-College of Veterinary Medicine for their provided facilities, which helped to improve the quality of this work.

\section{REFERENCES}

Abass Ghaidaa (2008): Epidemiological study for Toxoplasmosis andLeishmaniasis in stray dogs in Diwaniya city/Iraq. Kufa Journal For Veterinary Medical Science,4(2):3139.

Afkar M. Hadi and Azhar A.F. (2014): Role Of Domestic Cats Felis Catus As Reservoir Hosts Of Internal Parasites And Protozoa In Baghdad. Bulletin of the Iraq Natural History Museum,13(1): 89-94.

Afsaneh Amouei, Shahabeddin Sarvi, Mehdi Sharif, Sargis A. Aghayan Javad Javidnia, Azadeh Mizani, Mahmood Moosazadeh, Nemat Shams Seyed Abdollah Hosseini, Zahra Hosseininejad, Tooran Nayeri and Chegeni N. (2019): A systematic review of Toxoplasma gondii genotypes and feline Geographical distribution trends. Transbound Emerg Dis. 67:46-64. DOI: 10.1111/tbed.13340.
Aiz Noaman Naji (2016): Determination of Toxoplasma gondii lineages of sheep in Wasit, Iraq .Iraqi Journal of Veterinary Sciences 30(2): 23-26. DOI: 10.33899/ijvs.2016.121379.

Aldabagh, Muhammed Abdul-Gabar Hamoodi, Sadeq Kadhin Hachim, Khalid Waleed Qassim, Qasim Sharhan Al-Mayah, Jabbar Salman Hassan and Dunya Fareed Salloom (2014): Mmune profile in aborted Iraqi women with toxoplasmosis. Medical Journal of Babylon, 15 (1): 48-52

Al-Farwachi, M.; Basima Albadrani and AlHankawai, $O$ K. (2012): Detection of Toxoplasma gondii and Neospora caninum antibodies in cattle in Mosul city, Iraq. AL-Qadisiya Journal of Vet. Med. Sci.11(3): 46-49. DOI: 10.29079/vol11iss3art213.

Al Hamada Ali, Ihab Habib, Anne Barnes and Ian Robertson (2019): Risk Factors Associated With Seropositivity to Toxoplasma Among Sheep and Goats in Northern Iraq Vet Parasitol Reg Stud Reports 15:100264. doi: 10.1016/j.vprsr.2019.100264.

Alonso Aguirre, A.; Travis Longcore; Michelle Barbieri; Haydee Dabritz; Dolores Hill; Patrice N. Klein; Christopher Lepczyk; Emily L. Lilly; Rima McLeod; Judith Milcarsky; Caroline E. Murphy; Chunlei Su; Elizabeth VanWormer; Robert Yolken and Grant C. Sizemore (2019): The One Health Approach to Toxoplasmosis: $\quad$ Epidemiology, Control, and Prevention Strategies. EcoHealth.16, p: 378-390. doi.org/10.1007/s10393-019-01420-8.

Al-Taie Lazem H. and Shadan H. Abdulla (2011): Seroprevalance of Toxoplasmosis in sheep and goat: Iraq/ Sulaimania. Iraqi Journal of Veterinary Medicine. $\quad 35(1)$. DOI: https://doi.org/10.30539/iraqijvm.v35i 1.599

Alshahery, M.N. and Mansour, R.S. (2012): Detection of Toxoplasma gondii antibodies in horses in Mosul, Iraq. 
Iraqi Journal of Veterinary Sciences 26: 39-41.

Alexandra; D. Switzer; Audrey C. McMillanCole; Rickie W. Kasten; Matthew J. Stuckey; Philip H. Kass and Bruno B. Chomel (2013): Bartonella and Toxoplasma Infections in Stray Cats from Iraq. Am J Trop Med Hyg, 89(6): 1219-1224. doi: 10.4269/ajtmh.130353.

Al-Saidya, A.M.A. and AL-Kennany, E.R. (2006): Pathological And Histochemical Study On Cats And Mice Experimentally Infected With Toxoplasma Gondii. Iraqi Journal of Veterinary Science. 20(2): 249-263.

Anthea Brennan (2015): Toxoplasma gondii infection in Australian felines Msc thesis, University of Sydney, pp: 3335.

Asal Shoob Naser and Ibrahim A. Al Zubaidy (2016): Seroprevelance study of Toxoplasma gondii in horses and camels animal in Wasit province. Iraqi Journal of Veterinary Medicine, 40(1): DOI:

https://doi.org/10.30539/iraqijvm.v40i 1.152

Bethânia Ferreira Bastos, Beatriz Brener, Liza Gershony, Liliane Willi,Norma Labarthe, Cássia Pereira, and Flavya Mendes-De-Almeida

(2014):

Eroprevalence Of Toxoplasma Gondii (Nicole \& Manceaux, 1909) And Retroviral Status Of Client-Owned Pet Cats (Felis catus, Linnaeus, 1758) IN RIO DE JANEIRO, BRAZIL. Rev Inst Med Trop Sao Paulo, 56(3): 201-203. doi: $10.1590 / \mathrm{S} 0036-$ 46652014000300004

Cenci-Goga, B.T.; Paul V. Rossitto; Paola Sechi; Cheryl M.E. McCrindle; James S. Cullor (2011): Toxoplasma in Animals, Food, and Humans: An Old Parasite of New Concern. Foodborne Pathog Dis, 8(7):751-62. doi: 10.1089/fpd.2010.0795.

Chaichan, P. (2018): Epidemiology of Toxoplasma gondii in Thailand, Thesis in parasitology, University of Limoges, Thailand, PP: 15-20.
Dabritz Haydee, A.; Melissa A Miller; E Robert Atwill; Ian A Gardner; Christian M Leutenegger; Ann C Melli and Patricia A Conrad (2007): Detection of Toxoplasma Gondii-Like Oocysts in Cat Feces and Estimates of the Environmental Oocyst Burden. J Am Vet Med Assoc, 1; 231(11): 167684. doi: 10.2460/javma. 231.11.1676

Daland Christian Herrmann (2012):

Molecular typing of Toxoplasma gondii isolates from cats and humans in Germany. Dissertation. der Humboldt Universität zu Berlin,Germany.:16-18.,

Dass, S.A.; Anand Vasudevan; Deborah Dutta; Linda Jing Ting Soh; Robert Morris Sapolsky and Ajai Vyas (2011): Protozoan Parasite Toxoplasma Gondii Manipulates Mate Choice in Rats by Enhancing Attractiveness of Males. PLoS One, 6(11): 27229. doi: 10.1371/journal.pone.0027229

Dhamraa, R. Jalil and Mohammed, J. Alwan (2014): Seropathological Diagnosis of Toxoplasma gondii in Stray Cats in Baghdad Province. Iraqi Journal of Veterinary Medicine, (38(1): 92-98.

Dolores E Hill and Dubey, J P. (2016): Toxoplasma Gondii as a Parasite in Food: Analysis and Control. Microbiol Spectr4(4). doi: 10.1128/micro biolspec.PFS-0011-2015.

Dubey, J.P. and Beattie, C. (1988): Toxoplasmosis of Animals and Man. Parasitology, 100(3): 500-501. doi.org/10.1017/S0031182000078914

Dubey, J.P. and Jones, J.L. (2008): Toxoplasma gondii infection in humans and animals in the United States. International Journal for Parasitology 38 (2008) 1257-1278. doi:10.1016/j.ijpara.2008.03.007.

Dubey, $J$ P.; Sundar, N.; Hill, D.; Velmurugan, $G$ V.; Bandini, $L$ A.; Kwok, $O \mathrm{C} \mathrm{H.;} \mathrm{Majumdar,} \mathrm{D.} \mathrm{and} \mathrm{Su}$, $C$. (2008): High Prevalence and Abundant Atypical Genotypes of Toxoplasma Gondii Isolated From Lambs Destined for Human Consumption in the USA. Int $\mathrm{J}$ 
Parasitol38(8-9):999-1006.

doi: 10.1016/j.ijpara.2007.11.012.

Dubey $J$ P, Velmurugan, $G$ V.; AlvaradoEsquivel, C.; Alvarado-Esquivel, D.; Rodríguez-Peña, S.; Martínez-García, S.; González-Herrera, A.; Ferreira, L R.; Kwok, $O$ C H. and Su, C. (2009): Isolation of Toxoplasma Gondii From Animals in Durango, Mexico. J Parasitol, 95(2): 319-22. doi: 10.1645/GE-1874.1.

Edwards, J F. and Dubey, J P. (2013): Toxoplasma Gondii Abortion Storm in Sheep on a Texas Farm and Isolation of Mouse Virulent Atypical Genotype T. Gondii From an Aborted Lamb From a Chronically Infected Ewe. Vet Parasitol, 18; 192(1-3): 129-36. Doi: 10.1016/j.vetpar.2012.09.037

Eman Abdulla Qazaz and Azhar Ali Faraj (2016): $\quad$ Seroprevalence of Toxoplasmosis in Goat in Baghdad governorate. MRVSA 5 (2), 58-66.

Falah Hasan Hadi (2018): Incidence Study For Toxoplasma gondii Depending On Molecular And Immunological Characterization In Recurrent Aborted Women. Msc thesis, University of AlQadisiyah / College of Medicine Department of Microbiology, Iraq: 3444.

Fancourt, B.A. and Jackson, R.B. (2014): Regional seroprevalence of Toxoplasma gondii antibodies in feral and stray cats (Felis catus) from Tasmania. Australian Journal of Zoology 62, 272-283. doi: 10.1071/ZO14015

Ferguson, $D$ J.; Hutchison, $W M$. and Pettersen, E. (1989): Tissue Cyst Rupture in Mice Chronically Infected With Toxoplasma Gondii. An Immunocytochemical and Ultrastructural Study. Parasitol Res, 75(8): 599-503. doi: 10.1007/BF00930955

Flegr, J. (2013): Influence of latent Toxoplasma infection on human personality, physiology and morphology: pros and cons of the Toxoplasma-human model in studying the manipulation hypothesis. The Journal of Experimental Biology 216, 127-133. doi:10.1242/jeb.073635.

Hanon Basim Mohammed (2017): seroprevalence of brucellosis and toxoplasmosis in camels of Wasit Province, Iraq. Al-Qadisiyah Journal of Veterinary Medicine Sciences, 16(2): 29-32.

Hemdad H Mawlood; Wijdan M S Mero; Younis A A Bapeer and Chunlei, Su. (2019): Genotyping of Toxoplasma gondii from Stray Cats by Multilocus PCR-RFLP Techniques in Erbil city Kurdistan Region/Iraq. Journal of Physics: Conf. Series 1294, doi:10.1088/17426596/1294/6/062063

Hill, D. and Dubey, $J$ P. (2002): Toxoplasma Gondii: Transmission, Diagnosis and Prevention. Clin Microbiol Infect, 8(10): 634-40. doi: 10.1046/j.1469-0691.2002.00485.x.

Huan Ding; Yu-Meng Gao,; Yao Deng; Poppy H.L. Lamberton and Da-Bing, Lu. (2017): A systematic review and meta-analysis of the seroprevalence of Toxoplasma gondii in cats in mainland China. Parasites \& Vectors, 10(27): 410.

Janina Karla dela Cruz Advincula, Samira Yaser Perez Iewida, Cristina Cabanacan-Salibay (2010): Serologic detection of Toxoplasma gondii infection in stray and household cats and its hematologic evaluation. Scientia Medica (Porto Alegre), 20(1): 76-82.

Joanne $P$ Webster (2012): Review of "Toxoplasmosis of Animals andHumans (Second Edition)"by J.P. Dubey Parasites \& Vectors 3(1):1-2 DOI: $10.1186 / 1756-3305-3-112$.

Kader, J.M. and Al-Khayat, Z.A.Y. (2013): Serodiagnosis of toxoplasmosis in sheep and goats in Erbil city, Iraq. Iraqi Journal of Veterinary Sciences, 27(1): 21-23.doi. 10.33899/ijvs.2013.82947.

Karen Shapiro Lillian Bahia-OliveiraBrent Dixon Aurélien DumètreLuz A. De 
WitElizabeth Van WormerIsabelle Villena (2019): Environmental transmission ofToxoplasma gondii: Oocysts inwater, soil and food. Food and Waterborne Parasitology,15:1-10. https://doi.org/10.1016/j.fawpar.2019.e 00049

Kolören, Z. and Dubey, J.P. (2020): A review of toxoplasmosis in humans and animals in Turkey. Parasitology, 147(1): 12-28. DOI: https://doi.org/10.1017/S00311820190 01318

Majda Laboudi (2017): Review of toxoplasmosis in Morocco: seroprevalence and risk factors for toxoplasma infection among pregnant women and HIV- infected patients. Pan Afr Med J. 27: 269. doi: 10.11604/pamj.2017.27.269.11822.

Mikail, F.B. and Al-Barwary, L.T.O. (2014): Seroprevalence of toxoplasmosis in aborted ewes by using different immunologic tests in Duhok governorate, Kurdistan region, Iraq. Iraqi Journal of Veterinary 28, (1): 1115.

Mohammad T. Rahimil; Ahmad DaryaniI, Shahabeddin Sarvil; Azar ShokriI, Ehsan AhmadpourI; Saeed $H$. TeshnizilV; Azade MizaniI and Mahdi SharifI (2015): Cats and Toxoplasma gondii: A systematic review and metaanalysis in Iran. j. vet. res.82 (1): 6-11. http://dx.doi.org/10.4102/OJVR.V82I1 .823

Muhammad Hanafiah, Joko Prastowo, Sri Hartati, Dwinna Aliza and Raden Wisnu Nurcahyo (2018): Detection of Toxoplasma gondii copro-prevalence by polymerase chain reaction using repetitive 529 bp gene in feces of pet cats (Felis catus) in Yogyakarta, Indonesia. Veterinary World, 11(22): 1338-1344.

Muhannad Shweash, Noor N Al-Hayani, Hatem M. Hadeed and Thulfiqar Fawwaz Mutar (2018): Genotyping And Dna Sequencing Divergence Of Toxoplasma Gondii Strains Associated
With Rabbit Toxoplasmosis, Iraq, Int.

J. Life sci. Pharma res. 8(3): 24-34

Mulisa Megersa Kebeta (2014): Toxoplasma Gondii In Selected Sites Of Central Ethiopia: Seroprevalence, Risk Factors And Bioassay In Pigs. Msc Thesis, College of Veterinary Medicine and Agriculture of Addis Ababa,Addis Ababa University.pp:20-25.

Nawzat A. Issa (2017): I Nfection Rate Of Toxoplasmosis In Angora Goats Of Duhok Province-Iraq. Bas. J. Vet. Res. Vol. 16, No.2

Saad Al-Rubaie; Al-Jeburii Kefah and Taha, $O$ S. (2014): Serosurvillance on Toxoplasmosis in Camels (Camelus dromedarius) at Al-Najaf Province. Alnajaf For Veterinary Medical Sciences, 5(2): 204-210.

Sakban, F.M. and A'aiz, N.N. (2020): Investigate the Toxoplasma gondii infection in the consumed beef in AlDiwaniyah province. Iraqi Journal of Veterinary Sciences, 34(1): 95-99.

Saleem Amin Hasso (2007): A Review Of Confirmed Pathogen Of Dogs And Cats In Iraq. Bas, J, Vet, Res, 6(2): 156-162.

Silvia Maria Spalding (2005): I, II; Maria Regina Reis Amendoeira III; Carlos Henrique KleinIV; Luis Carlos Ribeiro. S erological screening and toxoplasmosis exposure factors among pregnant women in South of Brazil. Rev. Soc. Bras. Med. Trop. 38 (2):17$20 . \quad$ doi.org/10.1590/S003786822005000200009

Uzal, FA.; Plattner, BL. and Hostetter, JM. (2016): Alimentary system. In: Maxie MG, ed. Jubb, Kennedy, and Palmer's Pathology of Domestic Animals. Vol 2. 6th ed. Philadelphia, PA: Elsevier; 236-238.

Parameswaran, N.; R.M. O'Handley, B.M.E. Grigg, C.A. Wayne, D. and Thompsona, R.C.A. (2009): Vertical transmission of Toxoplasma gondii in Australian marsupials. Parasitology, 136(9): 939-944. doi: $10.1017 /$ S0031182009006453. 
Patrick Leo Taggart (2019): Ecology of Catborne Parasitoses in Australia. Doctor of Philosophy, School of Animal and Veterinary Sciences Faculty of Sciences The University of Adelaide. P: 20-22.

Vyas Ajai (2015): Mechanisms of Host Behavioral Change in Toxoplasma gondii Rodent Association. PLoS
Pathog.; 11(7): 1004935 . doi: 10.1371/journal.ppat.1004935.

Ziadoon Sameer, Z.; Alkafagi, N. and Nadem Ghazal (2017): Rate Of Infections With Toxoplasmosis In Baquba City, Diayala, Iraq. International Journal of Current Research, 9(4): 49081-49084 